\title{
Large-scale parallel finite element analysis of interface failure in CFRP models
}

\author{
Taichi Yamaguchi ${ }^{1 *}$, Gaku Hashimoto ${ }^{1}$, Hiroshi Okuda ${ }^{1}$ \\ ${ }^{1}$ Graduate School of Frontier Sciences, The University of Tokyo \\ ${ }^{\star}$ tyamaguchi@s.h.k.u-tokyo.ac.jp
}

Received: September 16, 2019; Accepted: December 26, 2019; Published: February 13, 2020

\begin{abstract}
Carbon fiber reinforced plastic composites (CFRP) exhibit complex failure mechanisms because they include fiber-matrix and interlaminar interfaces. Detailed modeling of fibers, matrices, and interfaces for conducting accurate progressive damage analysis requires large-scale finite element models. We developed parallel finite element analysis software, that can simulate failure of large-scale CFRP models including interfaces using cohesive elements. We verified the software comparing with proven commercial software. In addition, we validated the software using double cantilever beam tests. The simulation result agrees well with the experimental one. The software can analyze progressive interface damage of large-scale models with more than 77 million degrees of freedom.
\end{abstract}

Keywords: Parallel FEM, Large-scale, Cohesive element, Progressive damage analysis, CFRP

\section{Introduction}

Carbon fiber reinforced plastic composites (CFRP) are currently used for aircraft applications because of their excellent specific strength and specific elasticity. However, CFRPs exhibit highly complex interactive failure mechanisms because they include fiber-matrix interfaces and interlaminar interfaces as shown in Fig. 1, so experimental evaluation is essential. About 10,000 tests of material speciments are required to certificate an airframe structure [1]. To reduce the number of the experiments, simulation methods for failure analsys of CFRP have been developed [2-4]. It is necessary to model all these damages to accurately predict the damage of CFRP laminates. However, problems occur with large-scale models. To address this, in recent years, finite element method software capable of large-scale structural analysis has been developed along with the improvement in computer performance. One such large-scale parallel finite element analysis software is FrontISTR [5]. It can analyze large-scale problems by parallel computation based on domain decomposition. It can be used in systems ranging from personal 


\section{Journal of Advanced Simulation in Science and Engineering}

computers to supercomputers. It was developed in a national project in Japan, and is under continuous development and maintenance. Large-scale analysis cases with hundreds of millions of degrees of freedom using FrontISTR have been reported thus far [6, 7]. In addition, since it is open source and released as an MIT license, customization, such as adding new functions according to needs, can be performed without cost.

We aim to develop finite element software that can analyze large-scale models of CFRP that can model all kind of damages in detail by using FrontISTR as a seed program. In this study, as the first step, we have focused on the analysis of damage propagation at the interface, which is mostly observed and characteristic damages of composite materials. First, we implement a cohesive element that can model interface damage. This element is used for damagepropagation analysis of laminated interfaces [8-10]. It can also be used for damage-development analysis between the fiber and matrix [11-13] in the simulation of microbond tests [14]. Second, we verify the software comparing with proven commercial software. Finally, we validate the software conducting a simulation for the double cantilever beam (DCB) test, which is used to determine the mode I fracture toughness value of the laminated interface. Damageprogression analysis has been known to be affected by mesh size. The influence of mesh size is evaluated in the simulation of a DCB test using a two-dimensional model [9]. In this study, we change the mesh size from a small-scale to a large-scale model (up to 76.5 million degrees of freedom) in three dimensions analysis. We compare simulation results with experimental ones. We use automatic increment and consistent tangent stiffness in the Newton-Raphson method, unlike our previous work [15]. In addition, convergence performance is also evaluated.

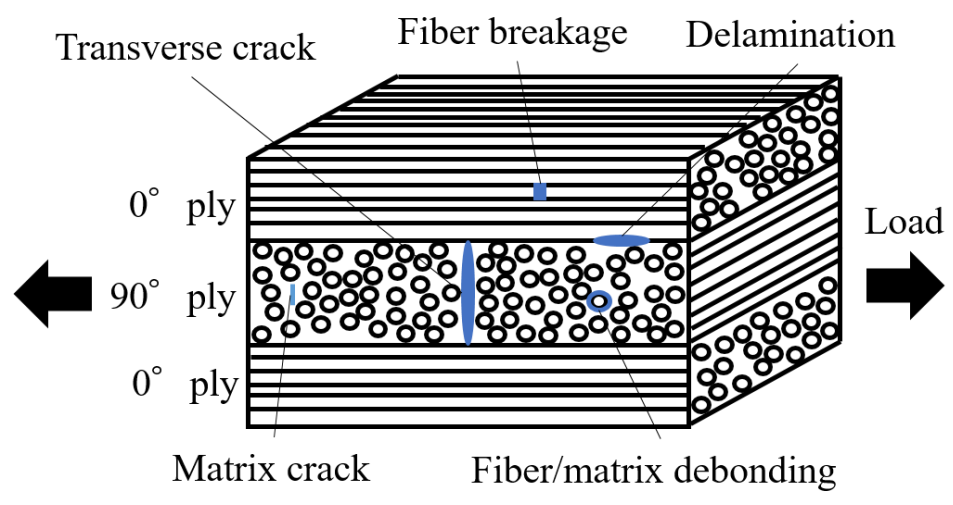

Figure 1: Failure of CFRP

\section{Formulation and implementation of interface damage model}

We use cohesive elements to model interface damage in CFRP. The formulation of the cohesive element [8] implemented into FrontISTR in this research is shown as follows. We use the formulation described in the reference [8], so the formulation is not new one but implementing this formulation to a large-scale parallel finite element analysis software is quite effective and crucial for analyzing CFRP failure. 


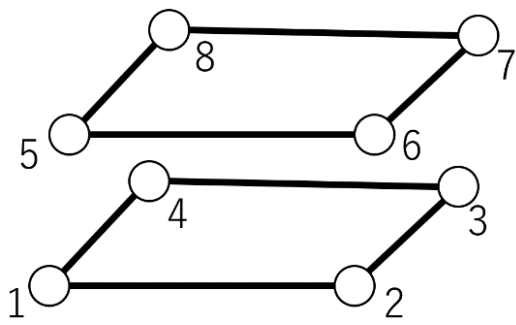

Figure 2: Topology of cohesive element

The topology of the cohesive element is shown in Fig. 2. The node numbers in the figure refer to the local node numbers in the element. The strain is determined from the relative distance between the lower surface consisting of nodes 1,2,3, and 4, and the upper surface consisting of nodes $5,6,7$, and 8 . We model the interface with zero initial thickness because the adhesive layer has a small thickness. The strain and the separation distance (displacement) of the interface have a same value by setting the layer thickness to one. The B-matrix (strain-displacement relationship) $\boldsymbol{B}$ is defined as follows.

$$
\begin{aligned}
& \boldsymbol{B}=
\end{aligned}
$$

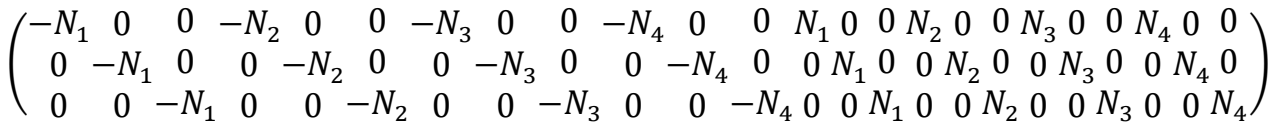

Here, $N 1, N 2, N 3$, and $N 4$ are shape functions of two-dimensional isoparametric elements. In the cohesive element, the orientation of the interface on the model is important, and thus, the compressive tension and shear directions of the interface are expressed in the local coordinate system. The displacement vector of the interface in the local coordinate system $\delta$ ' is obtained as follows.

$$
\begin{aligned}
\boldsymbol{\delta}^{\prime} & =\boldsymbol{P} \boldsymbol{B} \boldsymbol{u} \\
& =\boldsymbol{B} \boldsymbol{u}^{\prime}
\end{aligned}
$$

Where $\boldsymbol{\delta}^{\prime}$ has three components, one displacement in the tension and compression direction and two displacements in the shear direction. We define the nodal displacement vector of the global coordinate system as $\boldsymbol{u}$, the nodal displacement vector of the local coordinate system as $\boldsymbol{u}^{\prime}$, and the coordinate transformation matrix from the global to the local coordinate system as $\boldsymbol{P}$.

We use the interface construction law based on continuum damage mechanics. The stress in each direction can be obtained from Eq. (3), (4), and (5).

$$
\begin{gathered}
\sigma_{n}^{\prime}=\left\{\begin{array}{rr}
(1-\varphi) K_{n}^{\prime}{ }_{n} \delta_{n}^{\prime} & \delta^{\prime}{ }_{n} \geq 0 \\
K^{\prime}{ }_{n} \delta^{\prime}{ }_{n} & \delta^{\prime}{ }_{n}<0
\end{array}\right. \\
\sigma^{\prime}{ }_{s}=(1-\varphi) K^{\prime}{ }_{s} \delta^{\prime}{ }_{s} \\
\sigma^{\prime}{ }_{t}=(1-\varphi) K^{\prime}{ }_{t} \delta^{\prime}{ }_{t}
\end{gathered}
$$




\section{Journal of Advanced Simulation in Science and Engineering}

We define the damage variable as $\varphi$. Tensile and compressive direction is denoted by $n$, and two shear directions are denoted by $s$ and $t$. We set the stress, stiffness, and displacement in the tensile and compressive direction as $\sigma_{n}^{\prime}, K_{n}^{\prime}$, and $\delta_{\mathrm{n}}^{\prime}$, respectively, and the stress, stiffness, and displacement in the two shear directions as $\sigma_{s}^{\prime}, \sigma_{t}^{\prime}, K_{s}^{\prime}, K_{t}^{\prime}, \delta_{s}^{\prime}$, and $\delta_{t}^{\prime}$, respectively. The stress-strain matrix in the local coordinate system $\boldsymbol{D}^{\prime}$ is as shown in Eq. (6).

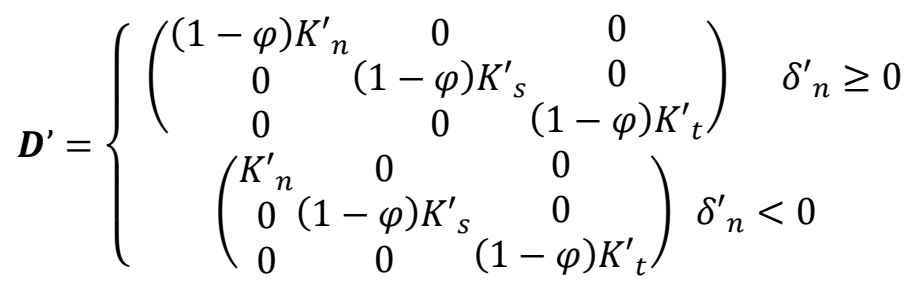

The damage variable $\varphi$ changes from 0 to 1 , where $\varphi=0$ indicates no damage, and $\varphi=1$ indicates destruction. Damage occurs when Eq. (7) holds.

$$
\left\{\frac{\left\langle\delta_{n}^{\prime}\right\rangle}{\delta_{n}^{o}}\right\}^{2}+\left\{\frac{\delta_{s}^{\prime}}{\delta_{s}^{o}}\right\}^{2}+\left\{\frac{\delta^{\prime} t}{\delta_{t}^{o}}\right\}^{2}=1
$$

We define the displacement when damage occurs in the tensile and two shear directions as $\delta_{n}^{o}, \delta_{s}^{o}, \delta_{t}^{o}$, respectively. The symbol $<>$ represents the Macaulay bracket. Here, the effective displacement $\delta_{m}$ is determined by Eq. (8).

$$
\delta_{m}=\sqrt{\left\langle\delta_{n}^{\prime}\right\rangle^{2}+\delta_{s}^{\prime 2}+\delta_{t}^{\prime 2}}
$$

The evolution rule of the damage variable $\varphi$ can be obtained from Eq. (9).

$$
\varphi=\frac{\delta_{m}^{f}\left(\delta_{m}^{\max }-\delta_{m}^{o}\right)}{\delta_{m}^{\max }\left(\delta_{m}^{f}-\delta_{m}^{o}\right)}
$$

The effective displacement at the onset of damage is $\delta_{m}^{o}$, the effective displacement at failure is $\delta_{m}^{f}$, and the maximum effective displacement at load history is $\delta_{m}^{\max }$. These relationships can be illustrated as shown in Fig. 3 if the characteristics do not differ in each direction. $K$ is the stiffness of the interface, $T$ is the interface strength, and the area of the triangle is the

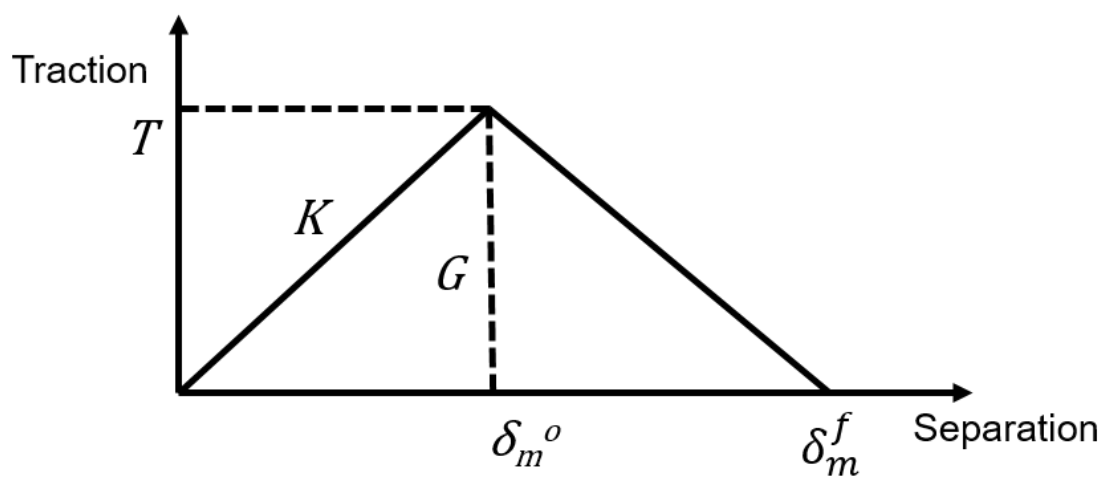

Figure 3: Interface properties 


\section{Journal of Advanced Simulation in Science and Engineering}

fracture toughness value $G$. In this case, we can parameterize the fracture properties of the interface with three values, for example, $K, T$, and $G$. Since analysis using cohesive elements has nonlinearity of damage development, we perform incremental analysis with the NewtonRaphson method. The element tangent stiffness matrix $\boldsymbol{K}_{\boldsymbol{e}}$ is obtained from Eq. (10) and (11), and the increment of the internal force vector of the element $\Delta \boldsymbol{Q}_{\boldsymbol{e}}$ is obtained from Eq. (12).

$$
\begin{gathered}
\boldsymbol{K}_{\boldsymbol{e}}=\left\{\begin{array}{c}
\int_{s} \boldsymbol{B}^{T} \boldsymbol{P}^{T} \boldsymbol{D}^{\prime} \boldsymbol{P} \boldsymbol{B} d s \quad \delta_{m}^{\max } \leq \delta_{m}^{o}, \delta_{m}^{f} \leq \delta_{m}^{\max } \\
\int_{s} \boldsymbol{B}^{T} \boldsymbol{P}^{T} \boldsymbol{D}^{\prime} \boldsymbol{P} \boldsymbol{B} d s+\boldsymbol{K}_{2 \boldsymbol{e}} \quad \delta_{m}^{o}<\delta_{m}^{\max }<\delta_{m}^{f}
\end{array}\right. \\
\boldsymbol{K}_{2 \boldsymbol{e}}=-\int_{s} \frac{\left(\delta_{m}-\delta_{m}^{\max }\right) \delta_{m}^{f} \delta_{m}^{o}}{\left(\delta_{m}^{\max }\right)^{2}\left(\delta_{m}^{f}-\delta_{m}^{o}\right)} \boldsymbol{B}^{T} \boldsymbol{P}^{T} \boldsymbol{\delta}^{\prime} \boldsymbol{\delta}^{\prime}{ }^{T} \boldsymbol{D}^{\prime} \boldsymbol{P} \boldsymbol{B} d s \\
\Delta \boldsymbol{Q}_{\boldsymbol{e}}=\int_{s} \boldsymbol{B}^{T} \boldsymbol{P}^{T} \boldsymbol{D}^{\prime} \boldsymbol{P} \boldsymbol{B} \Delta \boldsymbol{u} d s
\end{gathered}
$$

The area of the adhesive element is $\mathrm{s}$, and the incremental value of the nodal displacement vector in the global coordinate system is $\Delta \boldsymbol{u}$. Newton-Cotes numerical integration is used for these integrations.

\section{Accuracy verification of cohesive elements}

\subsection{Methods of verification}

We compare the results of FrontISTR with those of the commercial generic finite element software Abaqus to verify the accuracy of FrontISTR implemented with the cohesive element. We chose Abaqus for comparison because in the manual of Abaqus, reference [8] is cited and the same formulation as was implemented in FrontISTR is described. The model for verification is shown in Fig. 4. Cohesive elements are placed on the sides of the cylinder, which is divided into quarters, so that it can be applied to fiber-resin interfaces as well as laminated interfaces. We completely fix the top of the model, and give the end face of the cylinder imposed displacement (x-direction $0.5 \mathrm{~mm}$, y-direction $0.5 \mathrm{~mm}$, z-direction $0.5 \mathrm{~mm}$ ). The arrows in Fig. 4 indicate imposed displacement. Table 1 shows the interface material properties of the cohesive elements. Elements other than cohesive elements are divided into hexahedral primary elements. The Young's modulus and Poisson's ratio of these elements is $200 \mathrm{GPa}$ and 0.3 , respectively. These analysis conditions are common to FrontISTR and Abaqus. To examine the parallel computing performance based on domain division, we perform both serial and parallel computing 


\section{Journal of Advanced Simulation in Science and Engineering}

in the analysis using FrontISTR. The model is divided into two, four, and eight subdomains.

Figure 5 shows an example of decomposition into eight subdomains including the cohesive element as an example of domain decomposition.

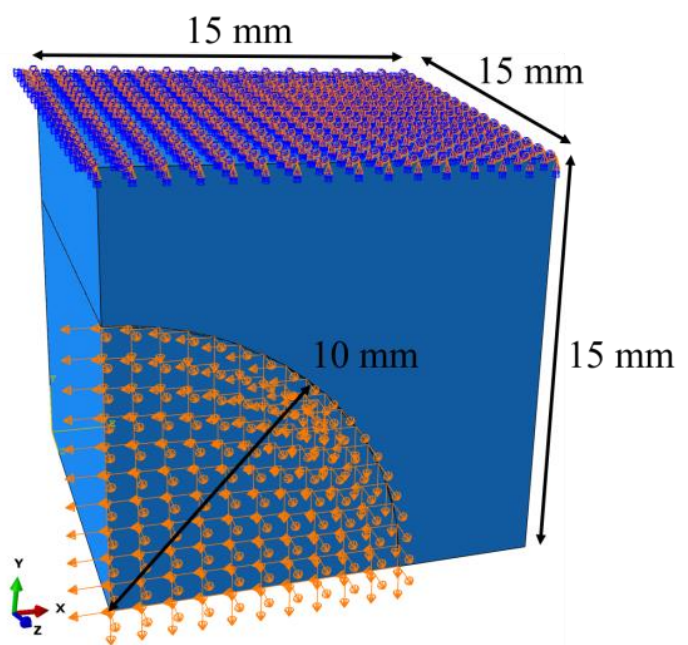

Figure 4: Evaluation model for cohesive elements

Table 1: Interface material properties for evaluation model

\begin{tabular}{ccccccc}
\hline $\mathrm{K}_{n}^{\prime}$ & $\mathrm{K}_{s}^{\prime}$ & $\mathrm{K}_{t}^{\prime}$ & $\delta_{n}^{o}$ & $\delta_{s}^{o}$ & $\delta_{t}^{o}$ & $\delta_{m}^{f}$ \\
\hline $1000 \mathrm{~N} / \mathrm{mm}^{3}$ & $100 \mathrm{~N} / \mathrm{mm}^{3}$ & $10 \mathrm{~N} / \mathrm{mm}^{3}$ & $0.5 \mathrm{~mm}$ & $0.3 \mathrm{~mm}$ & $0.4 \mathrm{~mm}$ & $0.5 \mathrm{~mm}$ \\
\hline
\end{tabular}

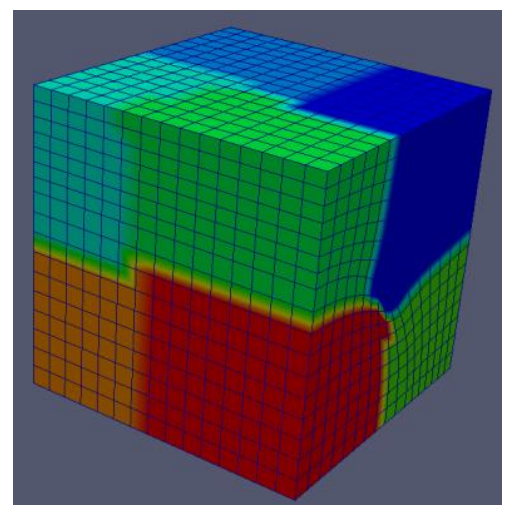

Figure 5: Domain decomposition of evaluation model

\subsection{Results of verification}

We chose two elements from the cohesive elements and compared the displacement, stress, and damage variables at the same integration point. Figure 6 shows the position of the cohesive elements used for comparison. Table 2 shows the comparison in element 4122, and Table 3 shows the comparison in element 4051 where the damage is further advanced. We chose element 4051 as the most damage progressed one and element 4122 as the moderate damage 
Journal of Advanced Simulation in Science and Engineering

progressed one in the simulation results. The color of the contour plot represents the value of the damage variable. The difference in the tables is the difference based on the Abaqus value. The results of FrontISTR and those of Abaqus agree with good accuracy. Table 4 shows the comparison results of serial and parallel calculations in element 4051. Parallel computing does not affect the analysis results. We confirmed that FrontISTR can perform interface-damage progress analysis with the same calculation accuracy as proven software, even in parallel calculation by domain decomposition.

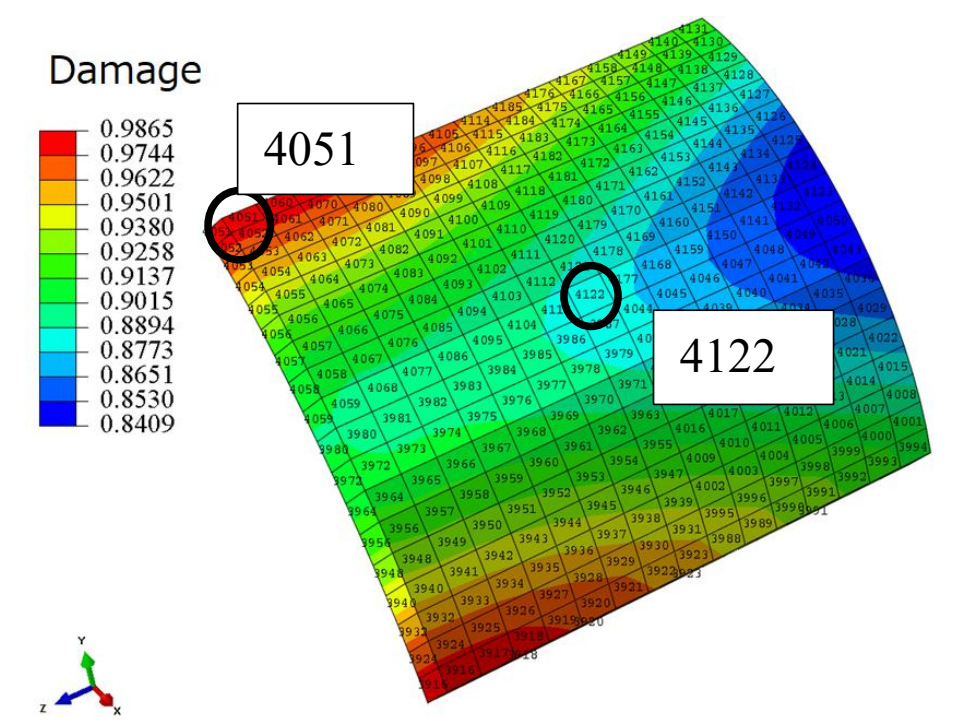

Figure 6: Color depicts values of $\varphi$ for comparision

Table 2: Comparison set of element 4122

\begin{tabular}{cccc}
\hline & FrontISTR & Abaqus & Difference [\%] \\
\hline$\delta_{n}^{\prime}[\mathrm{mm}]$ & 0.6658 & 0.6655 & 0.05 \\
$\delta_{s}^{\prime}[\mathrm{mm}]$ & 0.02591 & 0.02534 & 2.25 \\
$\delta_{t}^{\prime}[\mathrm{mm}]$ & 0.5222 & 0.5230 & 0.15 \\
$\sigma_{n}^{\prime}[\mathrm{MPa}]$ & 76.25 & 75.98 & 0.36 \\
$\sigma_{s}^{\prime}[\mathrm{MPa}]$ & 0.2967 & 0.2897 & 2.42 \\
$\sigma_{t}^{\prime}[\mathrm{MPa}]$ & 0.5981 & 0.5971 & 0.17 \\
Damage & 0.8855 & 0.8858 & 0.03 \\
\hline
\end{tabular}

Table 3: Comparison set of element 4051

\begin{tabular}{cccc}
\hline & FrontISTR & Abaqus & Difference [\%] \\
\hline$\delta_{n}^{\prime}[\mathrm{mm}]$ & 0.5221 & 0.5221 & 0.00 \\
$\delta_{s}^{\prime}[\mathrm{mm}]$ & 0.4754 & 0.4754 & 0.00 \\
$\delta_{t}^{\prime}[\mathrm{mm}]$ & 0.4997 & 0.5003 & 0.12 \\
$\sigma_{n}^{\prime}[\mathrm{MPa}]$ & 7.203 & 7.042 & 2.29 \\
$\sigma_{s}^{\prime}[\mathrm{MPa}]$ & 0.6559 & 0.6413 & 2.28 \\
$\sigma_{t}^{\prime}[\mathrm{MPa}]$ & 0.06893 & 0.06749 & 2.13 \\
Damage & 0.9862 & 0.9865 & 0.03 \\
\hline
\end{tabular}


Journal of Advanced Simulation in Science and Engineering

Table 4: Comparison set of serial and parallel analyses

\begin{tabular}{ccccc}
\hline & Serial & 2 MPI & 4 MPI & 8 MPI \\
\hline$\delta_{n}^{\prime}[\mathrm{mm}]$ & 0.5221 & 0.5221 & 0.5221 & 0.5221 \\
$\delta_{s}^{\prime}[\mathrm{mm}]$ & 0.4754 & 0.4754 & 0.4754 & 0.4754 \\
$\delta_{t}^{\prime}[\mathrm{mm}]$ & 0.4997 & 0.4997 & 0.4997 & 0.4997 \\
$\sigma_{n}^{\prime}[\mathrm{MPa}]$ & 7.203 & 7.203 & 7.203 & 7.203 \\
$\sigma_{s}^{\prime}[\mathrm{MPa}]$ & 0.6559 & 0.6559 & 0.6559 & 0.6559 \\
$\sigma_{t}^{\prime}[\mathrm{MPa}]$ & 0.06893 & 0.06893 & 0.06893 & 0.06893 \\
Damage & 0.9862 & 0.9862 & 0.9862 & 0.9862 \\
\hline
\end{tabular}

\section{Validation of the software simulating DCB tests}

\subsection{Methods}

We validated the software using DCB tests. We performed simulations of the DCB test shown in Fig. 7 using the developed software. The calculation model is a half-symmetric model. Two CFRP unidirectional plies, each with a thickness of $1.98 \mathrm{~mm}$, are stacked. The initial crack is $55 \mathrm{~mm}$, and a cohesive element is placed from the tip of the crack to the end of the specimen to model the interface. The parts other than the interface are divided into hexahedral primary elements. Table 5 [9] shows the orthotropic elastic properties of CFRP. Subscripts 1, 2, and 3 indicate the three orthogonal axes. Axis 1 is the fiber direction. E, G, and $v$ are the elastic modulus, shear modulus, and Poisson's ratio, respectively. The interface material properties are shown in Table 6 [9]. GIC, K, and T are the fracture toughness value in mode I, interface stiffness, and interface strength in the tensile direction, respectively. In this simulation, the fracture toughness value, interface stiffness, and interface strength in the shear direction are set to the same values as those in the tensile direction.

We simulate with mesh sizes of $1,0.5,0.25,0.125$, and $0.0625 \mathrm{~mm}$. The mesh shape is a rectangular solid. The length of the longitudinal side is set as the mesh size. The mesh size is denoted as $\mathrm{h}$. Forced positive and negative displacement in the z-direction can be applied to the top and bottom of the end face where the initial crack is located. The interface damage progression analysis is carried out until the crack opening displacement (COD) is $4.7 \mathrm{~mm}$. A half-symmetric model is used considering the symmetry of the model. We consider the value obtained by doubling the total value of the reaction force acting on the node given the imposed displacement as the load in the DCB test. The effect of mesh size on maximum load is evaluated.

The Newton-Raphson iterative calculation is used for geometrical nonlinearity and damage nonlinearity. The conditions for nonlinearity analysis are shown in Table 7. Increment size indicates the ratio of the displacement increment to the given imposed displacement. We evaluate the effect of mesh size on convergence performance.

The supercomputer system Oakforest-PACS [16] is employed. It consists of 8,208 nodes, and one node consists of 68 cores of CPU and a total of 112 GB of memory. In FrontISTR, MPI parallel calculation by subdomains and thread parallel calculation by OpenMP can be used as parallel computing. Table 8 shows the number of elements, number of nodes, degree of freedom (DOF), number of MPI and OpenMP in parallel, and number of nodes of OakforestPACS used for each condition. The domain decomposition is not performed for the model of $\mathrm{h}$ $=1 \mathrm{~mm}$. We use the CG method for the linear solver and use an AMG preconditioner. 


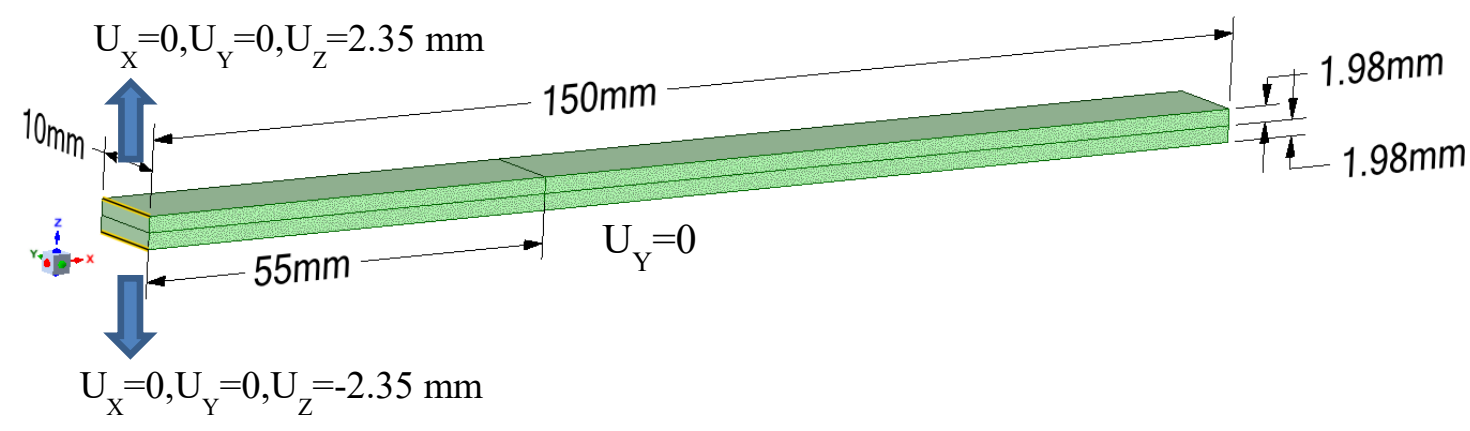

Figure 7: DCB model

Table 5: Mechanical properties for DCB simulation [9]

\begin{tabular}{cccccc}
\hline $\mathrm{E}_{11}$ & $\mathrm{E}_{22}=\mathrm{E}_{33}$ & $\mathrm{G}_{12}=\mathrm{G}_{13}$ & $\mathrm{G}_{23}$ & $v_{12}=v_{13}$ & $v_{23}$ \\
\hline $150.0 \mathrm{GPa}$ & $11.0 \mathrm{GPa}$ & $6.0 \mathrm{GPa}$ & $3.7 \mathrm{GPa}$ & 0.25 & 0.45 \\
\hline
\end{tabular}

Table 6: Interface material properties for DCB simulation [9]

\begin{tabular}{ccc}
\hline $\mathrm{G}_{\mathrm{IC}}$ & $\mathrm{K}$ & $\mathrm{T}$ \\
\hline $0.352 \mathrm{~N} / \mathrm{mm}$ & $10^{6} \mathrm{~N} / \mathrm{mm}^{3}$ & $60 \mathrm{MPa}$ \\
\hline
\end{tabular}

Table 7: Conditions of nonlinear analysis using Newton-Raphson

\begin{tabular}{lc}
\hline Increment size & 0.25 \\
\hline Convergence judgment & Residual norm $/$ inner power norm $<1.0 \times 10^{-6}$ \\
\hline Cut-back judgment & or \\
& Residual norm / inner power norm is NAN \\
\hline $\begin{array}{l}\text { Decreasing rate of increment } \\
\text { size due to cutback }\end{array}$ & 0.5 \\
\hline $\begin{array}{l}\text { Automatic increment judg- } \\
\text { ment }\end{array}$ & Substep of three or fewer Newton-Raphson iterations oc- \\
\hline
\end{tabular}

Increasing rate of increment

size due to automatic incre-

ment

Total increment size is 1.0

End judgment

or

Increment size $<1.0 \times 10^{-10}$ 
Journal of Advanced Simulation in Science and Engineering

Table 8: Calculation conditions

\begin{tabular}{lccccc}
\hline Mesh size $[\mathrm{mm}]$ & 1 & 0.5 & 0.25 & 0.125 & 0.0625 \\
\hline Number of elements & $6.95 \times 10^{3}$ & $5.18 \times 10^{4}$ & $3.99 \times 10^{5}$ & $3.13 \times 10^{6}$ & $2.48 \times 10^{7}$ \\
Number of nodes & $9.97 \times 10^{3}$ & $6.32 \times 10^{4}$ & $4.44 \times 10^{5}$ & $3.31 \times 10^{6}$ & $2.55 \times 10^{7}$ \\
DOF & $2.99 \times 10^{4}$ & $1.90 \times 10^{5}$ & $1.33 \times 10^{6}$ & $9.92 \times 10^{6}$ & $7.65 \times 10^{7}$ \\
MPI & - & 8 & 8 & 512 & 512 \\
OpenMP & 8 & 8 & 16 & 8 & 8 \\
Node of Oakforest-PACS & 1 & 1 & 2 & 64 & 64 \\
\hline
\end{tabular}

\subsection{Results and discussion}

The damage variable contour plot of the $\mathrm{h}=0.0625 \mathrm{~mm}$ model at a COD of $4.7 \mathrm{~mm}$ is shown in Fig. 8. The load-COD curve at each mesh size is shown in Fig. 9. Points indicate the load for each substep. In models other than $\mathrm{h}=1 \mathrm{~mm}$, parts with reduced load can be seen. The maximum load converges as the mesh size decreases. The maximum load at $\mathrm{h}=0.0625 \mathrm{~mm}$ is $62.6 \mathrm{~N}$ and the experimental value [8] is $62.5 \mathrm{~N}$. It is found that the simulation result agrees well with that of the experiment as the mesh size decreases. The distribution of damage variables at a COD of $4.4 \mathrm{~mm}$ is shown in Fig. 10. The damage distribution at $\mathrm{h}=0.125 \mathrm{~mm}$ is also similar to that at $\mathrm{h}=0.0625 \mathrm{~mm}$.

The time increment for each substep is shown in Fig. 11 to evaluate the convergence performance. The minimum increment size is $1.53 \times 10^{-5}$ for $\mathrm{h}=0.5 \mathrm{~mm}$ and $2.4 \times 10^{-4}$ for $\mathrm{h}=0.125$ $\mathrm{mm}$. It is found that these models have poor convergence performance. The minimum increment size of the $\mathrm{h}=0.0625 \mathrm{~mm}$ model is $3.13 \times 10^{-2}$, which indicates a good convergence performance. The calculation time and the number of iterations of Newton-Raphson for each mesh size are shown in Table 9. The degrees of freedom of the $\mathrm{h}=0.0625 \mathrm{~mm}$ model is nearly eight times that of $\mathrm{h}=0.125 \mathrm{~mm}$, but the total calculation time is approximately double. This is because $\mathrm{h}=0.0625 \mathrm{~mm}$ has good convergence performance and the number of iterations of Newton-Raphson is approximately one-fifth of that of $h=0.125 \mathrm{~mm}$. The convergence performance improves with decreasing mesh size. In large-scale analysis, iterative linear solvers are also essential. Table 10 shows the calculation time and number of iterations of the CG method in the first Newton-Raphson iteration for each mesh size. It is found that the number of iterations increases as the number of subdomains increases because the effect of preconditioning is reduced.

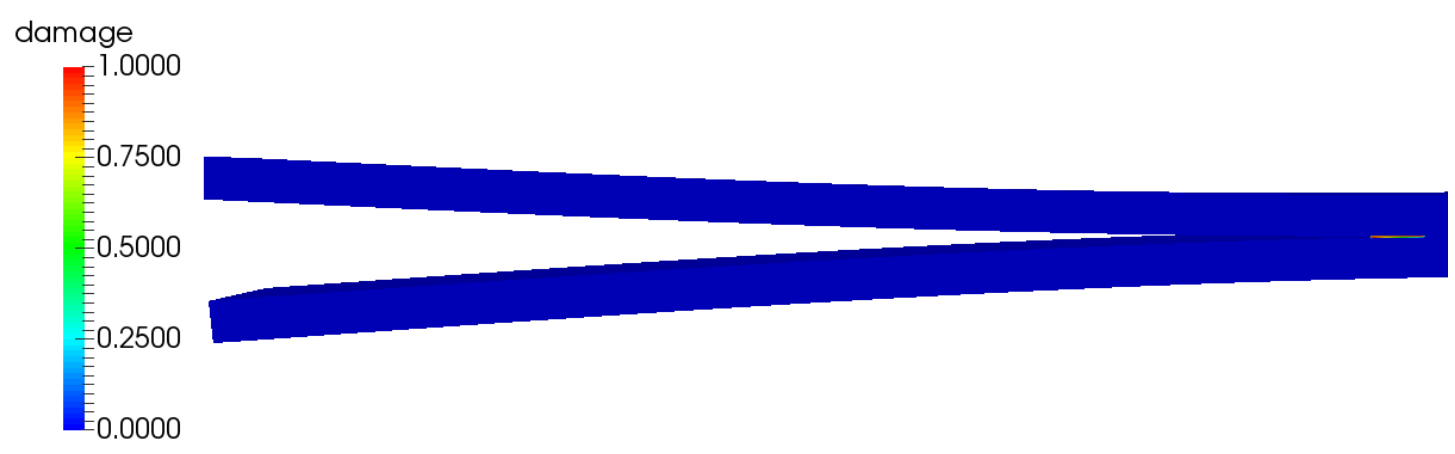

Figure 8: Damage variable contour of the $\mathrm{h}=0.0625 \mathrm{~mm}$ model at a COD of $4.7 \mathrm{~mm}$ 


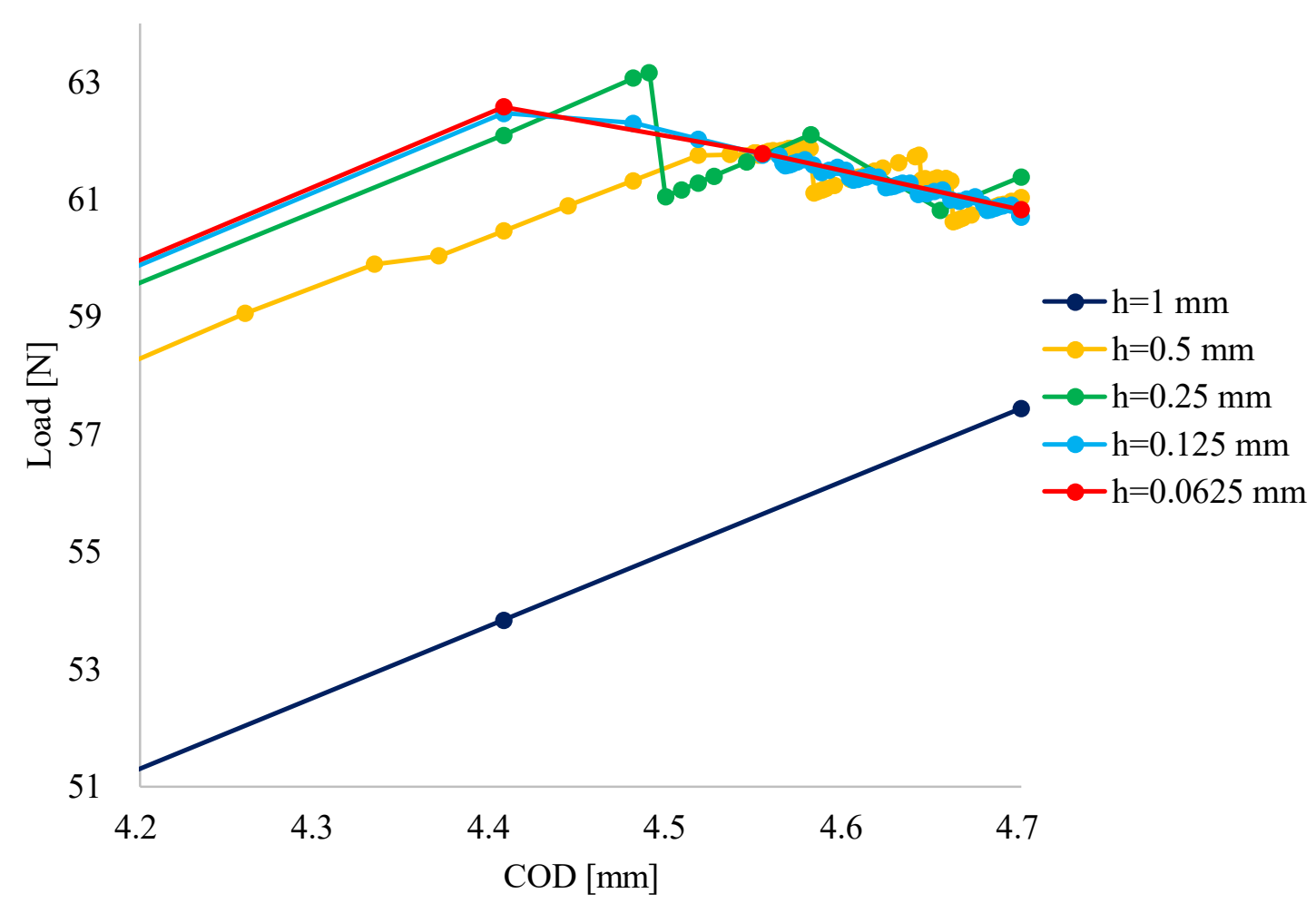

Figure 9: Load-COD curve (COD from 4.2 to $4.7 \mathrm{~mm}$ )

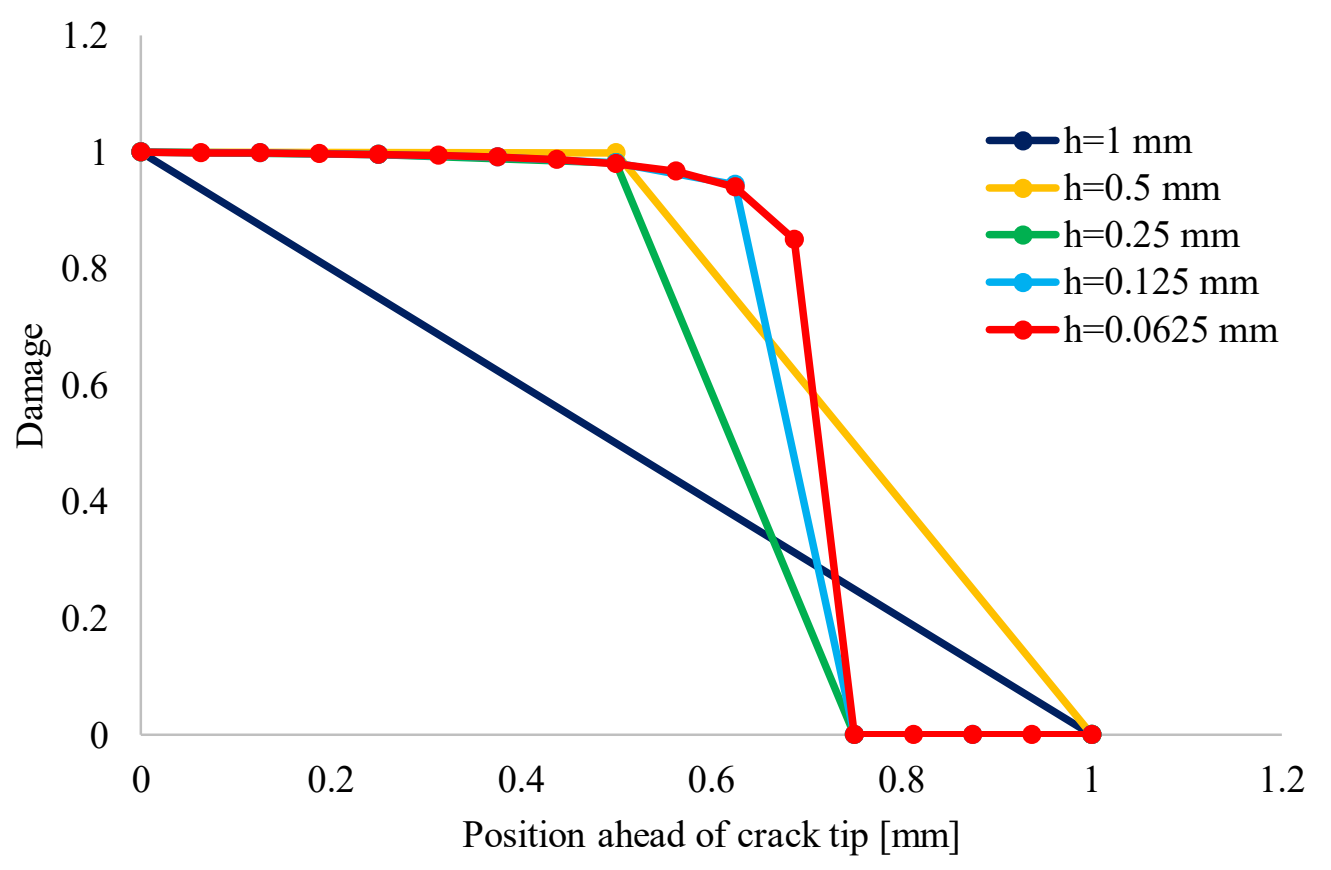

Figure 10: Distribution of damage ahead of the crack tip at $\mathrm{COD}=4.4 \mathrm{~mm}$ 


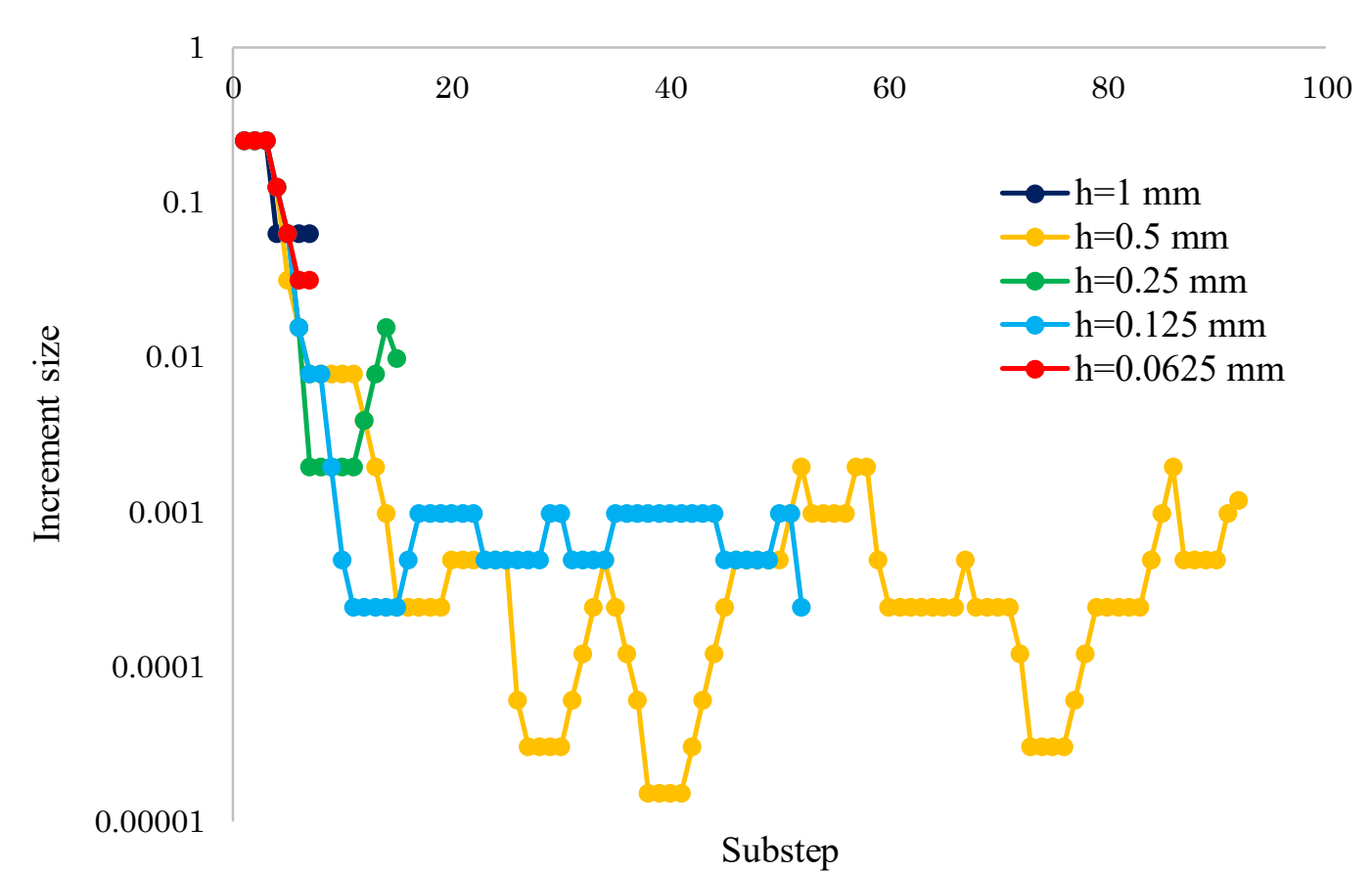

Figure 11: Increment size at each substep

Table 9: Calculation time and the number of iterations of Newton-Raphson

\begin{tabular}{cccccc}
\hline Mesh size & 1 & 0.5 & 0.25 & 0.125 & 0.0625 \\
\hline $\begin{array}{c}\text { Total Newton-Raphson itera- } \\
\text { tions }\end{array}$ & 60 & 1014 & 145 & 545 & 118 \\
$\begin{array}{c}\text { Total calculation time [s] } \\
\text { T. }\end{array}$ & 129 & 2,268 & 2,233 & 3,230 & 6,855 \\
\hline
\end{tabular}

Table 10: Calculation time and the number of iterations of CG method

\begin{tabular}{cccccc}
\hline Mesh size & 1 & 0.5 & 0.25 & 0.125 & 0.0625 \\
\hline $\begin{array}{c}\text { First CG method calculation } \\
\text { time [s] }\end{array}$ & 1.5 & 1.6 & 10.1 & 4.7 & 42.8 \\
$\begin{array}{c}\text { First CG method iterations } \\
\text { (1) }\end{array}$ & 85 & 84 & 89 & 160 & 275 \\
\hline
\end{tabular}

\section{Conclusions}

In this study, we developed parallel finite element analysis software that can analyze interfacedamage progression of large-scale CFRP models using cohesive elements. We verified the software comparing with proven commercial software and validated it using DCB tests. The results obtained in this study are as follows.

(1) We implemented a cohesive element in the open-source structural analysis software FrontISTR, and developed a software that can perform damage progressive analysis by parallel computation based on domain decomposition. 
Journal of Advanced Simulation in Science and Engineering

(2)We verified the software by comparing with commercial software. In the interface damageprogressive analysis, FrontISTR was able to obtain the same accuracy results as commercial software. It was also confirmed that the domain decomposition for parallel computing did not affect the analysis results.

(3) We validated the developed software by comparing with experimental results. The DCB test was simulated using the software. The maximum load converged for a sufficiently small mesh size, and the result agreed well with the experimental value [8].

(4) In the damage progression analysis, nonlinear analysis using the Newton-Raphson method was performed. By making the mesh sufficiently fine, it was observed that the convergence performance was improved.

\section{References}

[1] B. Cox, and Q. Yang, In quest of virtual tests for structural composites. Science, 314.5802 (2006), pp.1102-1107.

[2] B.N. Cox, S.M. Spearing, and D.R. Mumm, Practical challenges in formulating virtual tests for structural composites. Mechanical response of composites (2008), pp.57-75.

[3] J. LLorca, C. González, J.M. Molina-Aldareguía, J. Segurado, R. Seltzer, F. Sket, M. Rodríguez, S.Sádaba, R. Muñoz, and L.P. Canal, Multiscale modeling of composite materials: a roadmap towards virtual testing, Advanced Materials 23.44 (2011), 5130-5147.

[4] T. Okabe, Recent studies on numerical modelling of damage progression in fibre-reinforced plastic composites. Mechanical Engineering Reviews (2014), 14-00226.

[5] https://github.com/FrontISTR/FrontISTR (accessed 2019-09-13).

[6] H. Kadowaki, G. Hashimoto, H. Okuda, T. Higuchi, H. Jinnai, E. Seta, and T. Saguchi, Evaluation of the appropriate size of the finite element representative volume for filled rubber composite analyses, Mechanical Engineering Journal, 3, 5 (2016), 16-00372.

[7] Y. Ihara, G. Hashimoto, and H. Okuda, Web-based integrated cloud CAE platform for large-scale finite element analysis, Mechanical Engineering Letters, 3 (2017), 17-00520.

[8] P.P. Camanho, and C.G. Davila, Mixed-mode decohesion finite elements for the simulation of delamination in composite materials, NASA/TM-2002-211737,2002.

[9] A. Turon, C.G. Davila, P.P. Camanho, and J. Costa, An Engineering Solution for Mesh Size Effects in the Simulation of Delamination Using Cohesive Zone Models, Engineering fracture mechanics, 74, 10 (2007), pp. 1665-1682.

[10] V. Shanmugam, R. Penmetsa, E. Tuegel, and S. Clay, Stochastic modeling of delamination growth in unidirectional composite DCB specimens using cohesive zone models, Composite Structures, 102 (2013), pp. 38-60.

[11] M.A. Minnicino, and M.H. Santare, Modeling the progressive damage of the microdroplet test using contact surfaces with cohesive behaviour, Composites science and technology, 72.16 (2012), pp. 2024-2031.

[12] S. Sockalingam, M. Dey, J.W. Gillespie Jr, and M. Keefe, Finite element analysis of the 
Journal of Advanced Simulation in Science and Engineering microdroplet test method using cohesive zone model of the fiber/matrix interface, Composites Part A: Applied Science and Manufacturing, 56 (2014), pp. 239-247.

[13] M. Sato, E. Imai, J. Koyanagi, Y. Ishida, and T. Ogasawara, Evaluation of the interfacial strength of carbon-fiber-reinforced temperature-resistant polymer composites by the micro-droplet test, Advanced Composite Materials, 26, 5 (2017), pp. 465-476.

[14] S. Zhandarov, and E. Mader, Characterization of fiber/matrix interface strength: Applicability of different tests, approaches and parameters, Composites Science and Technology, 65 (2005), pp. 149-160.

[15] T. Yamaguchi, G. Hashimoto, and H. Okuda, Development of parallel finite element method software for analyzing progressive interface failure of large - scale CFRP models, in Proc. 22 $2^{\text {nd }}$ ICCM, Melbourne, (2019).

[16] https://www.cc.u-tokyo.ac.jp/en/supercomputer/ofp/service/ (accessed 2019-09-13). 